\title{
Characterizing Public Health Actions in Response to Syndromic Surveillance Alerts
}

\author{
Laura Rivera', Rachel Savage ${ }^{2}$, Natasha Crowcroft ${ }^{1,2}$, Laura Rosella ${ }^{2,1}$, Li Ye ${ }^{1,2}$, Shelly \\ Bolotin $^{1,2}$, Wendy Lou ${ }^{1}$ and lan Johnson ${ }^{\star 1,2}$
}

${ }^{1}$ Public Health Ontario, Toronto, ON, Canada; ${ }^{2}$ University of Toronto, Toronto, ON, Canada

\section{Objective}

To describe results of a prospective study to assess the impact of using a standard process by which public health units (PHUs) investigate syndromic surveillance alerts for respiratory illness.

\section{Introduction}

Public health in Ontario, Canada has no standardized system for carrying out syndromic surveillance. Previous research had demonstrated wide variation in the implementation of syndromic surveillance.

\section{Methods}

We recruited 15 PHUs that routinely accessed syndromic data (9 intervention, 6 control). Many already received alerts of aberrant events directly from their system. Both intervention and control groups were encouraged to continue standard practices to receiving and responding to these alerts, but in addition, intervention PHUs received alerts from a standard statistical algorithm designed by the study team to maximize specificity, and were asked to implement an evidence-based protocol for investigating all alerts. Data collection forms ("logbooks") collected qualitative and quantitative information about the alerts and follow-up. Logbook data were grouped into themes, and tabulated to determine how frequently they occurred.

\section{Results}

Between October 2013 to February 2015, 15 PHUs received 1,969 alerts for respiratory and influenza-like illness syndromes from emergency department visit data. Of these alerts, 942 alerts were for the intervention units and 1,027 were for the control health units. Two hundred and twelve $(24 \%)$ ) of the intervention alerts were generated by the study. PHUs in the intervention group checked alerts three times more frequently than control health units for alternate explanations as specified in the protocol. Control health units performed $20 \%$ more epidemiological investigations of aberrant events they received. Figure 1 illustrates the types of actions taken. For control health units, $549(53 \%)$ of the alerts were deemed to warrant a response but 341 (33\% of all alerts) of these responses were described as "warranting a response by watchful waiting". In contrast, for intervention health units the numbers were 165 (18\%) and 119 (13\%) respectively. Overall, less than $10 \%$ of the alerts led to warranting a response by internal and external notifications. The high percentage of alerts warranting a response in the control group is partially due to one control health unit having a low threshold for designated that action was warranted. Next steps include adjusting for repeated measures by health units in calculating statistical significance.

\section{Conclusions}

The use of a standard protocol appears to have altered the approach to verification and validation of alerts. However, the large number of alerts translated into few tangible public health actions. Syndromic surveillance of emergency department visits appeared mainly to be used for "watchful waiting" and situational awareness.
Figure 1. Percent of alerts to which a response was taken

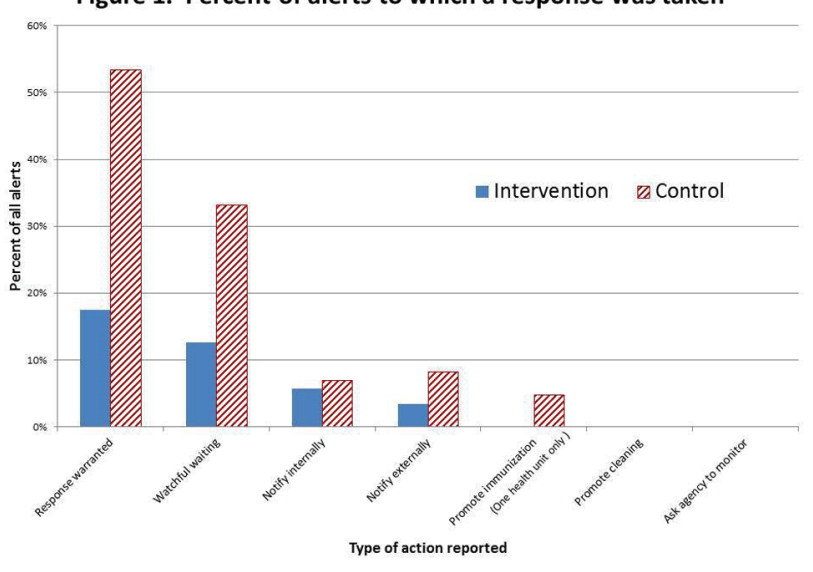

Keywords

Syndromic surveillance; Evaluation; Response; Public Health

\section{Acknowledgments}

We wish to acknowledge the participating health units and epidemiologists: Algoma Public Health, Brant Public Health, Halton Public Health, Hamilton Public Health, KFLA Public Health, Lambton Public Health, Leeds-Grenville Lanark Public Health, Niagara Public Health, North Bay Public Health, Ottawa Public Health, Peterborough Public Health, Peel Public Health, Simcoe-Muskoka Public Health, Sudbury District Health Unit, and Toronto Public Health

\section{References}

References

Chu A, Savage R, Whelan M, Rosella LC, Crowcroft NS, Willison D, Winter AL, Davies R, Gemmill I, Mucchal PK and Johnson I.

Chu A, Savage R, Willison D, Crowcroft NS, Rosella LC, Sider D, Garay J, Gemmill I, Winter AL, Davies R and Johnson I. The use of syndromic surveillance for decision-making during the H1N1 pandemic: A qualitative study. BMC Public Health 2012, 12:929 (30 October 2012) http://www.biomedcentral.com/1471-2458/12/929

\section{*lan Johnson}

E-mail: ian.johnson@oahpp.ca 